\title{
Antifungal activity of P3HB microparticles containing tebuconazole
}

\author{
Anna M. Shershneva ${ }^{a}$, Anastasiya V. Murueva ${ }^{\mathrm{b}}$, Natalia O.Zhila, ${ }^{\mathrm{a}, \mathrm{b}}$, Svetlana V. Prudnikova ${ }^{\mathrm{a}}$, Tatiana G. \\ Volova $^{\text {a,b }}$ \\ ${ }^{a}$ Siberian Federal University, Institute of Fundamental Biology and Biotechnology, 79 Svobodny, Krasnoyarsk, \\ 660041, Russia \\ ${ }^{b}$ Institute of Biophysics SB RAS, Federal Research Center "Krasnoyarsk Science Center SB RAS", 50/50 \\ Akademgorodok, Krasnoyarsk, 660036, Russia \\ *Corresponding author E-mail address: sheranna@inbox.ru \\ Tel.: +73912905491, Fax.:+73912433400
}

\begin{abstract}
In this study, tebuconazole (TEB)-loaded poly-3-hydroxybutyrate (P3HB)-based microparticles were developed and comprehensively characterized. TEB-loaded microparticles $(10,25$, and 50\%) were prepared using double emulsification technique. Encapsulation efficiency of TEB varied from 59 to $86 \%$. As the loading amount was increased, the average diameter of microparticles increased too, from 41.3 to $71.7 \mu \mathrm{m}$, while zeta potential was not influenced by TEB loading, varying between -32.6 and $-35.7 \mathrm{mV}$. TEB was released gradually from 10, 25, and $50 \%$ loaded particles, and over 60 days, 25,43 , and 38\%, respectively, of the initially loaded amount was released. The data obtained from in vitro TEB release were fitted to different mathematical models. It was shown that the release profiles of TEB could be best explained by Zero-order, Higuchi models and Hixson-Crowell. The antifungal activity of the $\mathrm{P} 3 \mathrm{HB} / \mathrm{TEB}$ microparticles against phytopathogenic fungi $F$. moniliforme and $F$. solani was comparable to that of the free fungicide. Thus, hydrophobic agrochemicals (TEB) can be effectively encapsulated into $\mathrm{P} 3 \mathrm{HB}$ microparticles to construct slow-release formulations.
\end{abstract}

Keywords: Poly-3-hydroxybutyrate, tebuconazole, encapsulation, microparticles, antifungal activity

\section{Introduction}

Over the past decade, uncontrolled use of pesticides in agriculture has caused bioaccumulation of agrochemicals in soil, leading to the loss of biodiversity and destroying habitats for living organisms [1]. Fungicides are pesticides used to control fungal diseases of plants by, e.g., inhibiting fungal spore germination. The commonly used fungicides are triazols (tebuconazole, hexaconazole, paclobutrazol, etc.). Preventative applications of triazol fungicides are more effective than curative applications.

In spite of the high efficacy of these fungicides, they have been found to produce a toxic effect on seed germinating capacity and inhibit plant growth [2-4]. The unfavorable effect occurs via the following mechanism: fungicides shift the balance of phytohormones in plant tissues and inhibit biosynthesis of gas, causing a temporary increase in the content of abscisic acid in plants [4-6]. Moreover, the wide use of agricultural fungicides poses potential contamination risk to both soil and aquatic ecosystems [1, 7-8].

Innovative approaches are needed to manage contaminated ecosystems. Fungicide toxicity to the environment can be reduced by using novel polymeric carriers enabling controlled release of the fungicide, which both increases the efficacy of agrochemicals and minimizes their adverse effects on the environment [9-11].

Polymeric microparticles can act as transport media for active substances and offer advantages including improved physical, chemical, and biological stabilities, simple and reproducible preparation, and applicability to a wide range of agrochemicals [12-15]. Also, microparticles may increase the efficacy of the agrochemicals, offering better results with lower doses and number of applications, as well as they may decrease the risk of environmental contamination and the toxicity to humans and other non-target organisms [16].

Considerable research effort in the last decade has gone into studying polyhydroxyalkanoates (PHAs) polyesters of alkanoic acids, produced by microorganisms, which, as well as polycaprolactone, polylactides, and polyglycolides, are used to construct systems enabling controlled release of the active ingredient. PHAs can be degraded into end products (aerobically to $\mathrm{CO}_{2}$ and $\mathrm{H}_{2} \mathrm{O}$ ) in different biological media, and this is one of their most valuable properties [17].

Research on embedding agrochemicals in PHA-based microparticles is still in its infancy. Some authors reported encapsulation of the herbicides atrazine and ametrine in microspheres prepared from $\mathrm{P} 3 \mathrm{HB} / 3 \mathrm{HV}$ [12, 18] and encapsulation of the pesticide malathion in microspheres prepared from poly-3-hydroxybutyrate blended with polycaprolactone [19]. A search of the available literature did not reveal any studies reporting the use of PHAs to construct slow-release fungicide formulations. The previous studies by our team described experimental tebuconazole formulations shaped as smooth films and 3D constructs and reported TEB release kinetics [20-21]. Experiments with two cultures, $F$. moniliforme and $F$. solani, proved that the experimental formulations of tebuconazole embedded in the P3HB matrix showed antifungal activity.

The purpose of this study was to construct and investigate polymeric microparticles based on poly-3hydroxybutyrate containing a fungicide - tebuconazole.

\section{Materials and Methods}

\subsection{Materials}


Polymer of $\beta$-hydroxybutyric acid (poly-3-hydroxybutyrate, $\mathrm{P} 3 \mathrm{HB}$ ) was used as a polymeric carrier for the fungicide. The polymer was synthesized at the Institute of Biophysics SB RAS using the technology developed by Volova et al. [22]. Systemic fungicide Raxil Ultra (Bayer CropScience, Russia) and its active ingredient chemically pure Tebuconazole (97\%, TEB) (Nanjing Zhongli New Material Technology Co., Ltd, China) - were used in this study (Fig. 1). TEB has a broad spectrum of activity. It provides effective protection against various diseases in cereals and helianthus. This fungicide disinfects seeds and partially decontaminates soil and plant residues around the seeds. The time of degradation in soil is 177 days.

\subsection{Preparation of controlled release formulation}

Microparticles were constructed using the emulsion technique. First, a polymer/TEB suspension was prepared by dissolving $200 \mathrm{mg}$ P3HB and 20, 50, and $100 \mathrm{mg}$ TEB (to achieve 10, 25, and 50\% loading, respectively) in $10 \mathrm{ml}$ organic solvent - dichloromethane. Then a double oil/water $(\mathrm{O} / \mathrm{W})$ emulsion was prepared by gradually pouring the solution into $100 \mathrm{ml}$ of a $1 \%$ polyvinyl alcohol solution (Mw 30-50 kDa, Sigma-Aldrich, U.S.). After that, the emulsion was agitated at $750 \mathrm{rpm}$ for $24 \mathrm{~h}$ using an MR Hei-Standard magnetic stirrer (Heidolph, Germany) to prepare micro-sized particles. Microparticles were collected by centrifuging using Centrifuge 5810 R, 5417 R (Eppendorf, Germany) (at $10000 \mathrm{rpm}$, for $7 \mathrm{~min}$ ), rinsed in distilled water, and freeze dried (Alpha 1-2 LD plus, Christ ${ }^{\circledR}$, Germany).

\subsection{Analysis of $\mathrm{P} 3 \mathrm{HB} / \mathrm{TEB}$ microparticles}

The size of the microparticles was determined using a system for quantitative and qualitative particle analysis - FlowCam (FluidImaging, U.S.); a Zetasizer Nano ZS (Malvern, U.K.) particle analyzer was used to determine zeta potential based on electrophoretic activity of microparticles.

Surface morphology of microparticles was examined using scanning electron microscopy with an S-5500 microscope (Hitachi, Japan) in the Joint Instrument Use Center at the Krasnoyarsk Scientific Center Siberian Branch of Russian Academy of Sciences. The samples were sputter-coated with platinum using a sputter coater K550X (Emitech, Quorum Technologies Ltd., U.K.).

The yield $(Y)$ of microparticles was calculated as percent of the mass of the polymer used to prepare them, with the following formula:

$$
Y=\left(W_{m} / W_{p}\right) * 100 \%
$$

where $W_{m}$ is the weight of the microparticles, $\mathrm{mg}$, and $W_{p}$ is the weight of the polymer used to prepare the microparticles, mg.

The amount of the fungicide embedded in the microparticles was determined using chromatograph/mass spectrometer 7890/5975C (“Agilent Technology”, U.S.); calibration was performed with fungicide solutions of known concentrations. The encapsulation efficiency (EE) was calculated as percent of the fungicide amount embedded in the matrix using the following formula:

$$
E E=\left(M_{w} / M_{e}\right) * 100 \% \text {, }
$$

where $M_{w}$ is the initial weight of the fungicide and $M_{e}$ is the weight of the fungicide encapsulated in the polymeric matrix.

\subsection{In vitro TEB release studies}

Kinetics of TEB release from the polymeric microparticles was studied in vitro in laboratory water systems as described elsewhere [23]. Samples of microparticles were sterilized and placed into 500-ml sterile conical flasks filled with sterile distilled water $(100 \mathrm{ml})$. The flasks were incubated at $25^{\circ} \mathrm{C}$ in an Innova 44 New Brunswick temperature controlled incubator shaker at $150 \mathrm{rpm}$. Samples for analysis were collected periodically, under aseptic conditions, and an aliquot of water was added to the flask to maintain a constant volume of liquid in it. TEB was extracted with chloroform three times to determine its concentration. The chloroform extracts were passed through sodium sulfate. Chloroform was removed in a rotary vacuum evaporator. The amount of TEB released $\left(A_{T E B}\right)$ was determined as percentage of the tebuconazole encapsulated in the polymer matrix, using the following formula:

$$
A_{T E B}=\left(R_{A} / E_{A}\right) \times 100 \% \text {, }
$$

where $E_{A}$ is the encapsulated amount, $\mathrm{mg}$, and $R_{A}$ is the amount released, $\mathrm{mg}$.

The data obtained from in vitro experiments were fitted to various mathematical models to assess the TEB release kinetics [24].

\section{Zero-order kinetic model}

$$
Q_{t}=Q_{0}+K_{0} * l
$$

where $\mathrm{Q}_{\mathrm{t}}$ is amount of the fungicide dissolved in time $t, \mathrm{Q}_{0}$ is initial amount of the fungicide in the solution, and $\mathrm{K}_{0}$ is zero-order release constant.

First-order kinetic model

$$
\log Q_{t}=\log Q_{0}+\frac{K_{1}+t}{2.303}
$$

where $\mathrm{Q}_{\mathrm{t}}$ is amount of the fungicide dissolved in time $\mathrm{t}, \mathrm{Q}_{0}$ is initial amount of the fungicide in the solution, and $\mathrm{K}_{1}$ is first-order release constant.

\section{Higuchi model}

The model relates cumulative release of the fungicide versus square root of time as shown in Eq. (3).

$$
Q=K_{H} * \sqrt{t}
$$




\section{Hixson-Crowell model}

This model relates cube root of the percentage of the fungicide remaining in microparticles versus time. As given by Eq. (4).

\section{Korsmeyer-Peppas model}

$$
Q_{0}^{1 / 3}=Q_{t}^{1 / 3}+K_{S} * t
$$

This model relates exponentially the fungicide release to the elapsed time. The equation is given as Eq. (5).

$$
\frac{Q_{l}}{Q_{\infty}}=K_{k} * \iota^{n}
$$

\subsection{Antifungal activity of P3HB/TEB microparticles}

The antifungal activity of $\mathrm{P} 3 \mathrm{HB}$ microparticles containing TEB was investigated in experiments with phytopathogenic fungi of the genus Fusarium ( $F$. moniliforme and $F$. solani), which were extracted from the field soil and grown on the malt extract agar (MEA, Sigma-Aldrich, U.S.) in Petri dishes at a temperature of $25^{\circ} \mathrm{C}$ for 5-7 days. Then, 5-mm diameter slabs of agar were aseptically drilled from the culture regions with actively growing colonies. A slab with the fungal culture and P3HB/TEB microparticles were placed at opposite sides of the Petri dish containing sterile MEA. The dishes were incubated in a thermostat at $25^{\circ} \mathrm{C}$ for $72 \mathrm{~h}$; then, we measured the radius of the fungal mycelium and determined the degree of fungus growth inhibition relative to the control. As a positive control $\left(\mathrm{Control}^{+}\right)$, we used the commercial TEB formulation (Raxil Ultra) at the same concentration; as a negative control, fungi were grown without TEB.

\subsection{Statistical analysis}

Statistical analysis of results was performed using the standard software package of Microsoft Excel, STATISTICA 8. Arithmetic means and standard deviations were determined using Student's $t$ test. Results are given as $\mathrm{X} \pm \mathrm{m}$.

\section{Results}

\subsection{Characterization of the microparticles}

Using the technique of solvent evaporation from emulsion, we produced microparticles with initial TEB loading of 10,25 , and $50 \%$ of the polymer weight.

To prepare TEB-loaded microparticles, we dissolved the fungicide and the polymer in dichloromethane. Although TEB solubility in dichloromethane is higher than $200 \mathrm{~g} / \mathrm{L}\left(\right.$ at $\left.20^{\circ} \mathrm{C}\right)$ and its molecular weight is low $307.8 \mathrm{~g} / \mathrm{mol}$., analysis showed formation of large undissolved TEB crystals on the surface of microparticles (Fig. 2). The most likely explanation for this is that TEB concentration was high and it did not dissolve completely because of the presence of the high-molecular-weight polymer chains of P3HB in the solution. As the TEB loading was increased from 10 to $50 \%$, the amount of TEB crystals on the surface of microparticles increased too.

Moreover, as the TEB loading was increased, the average diameter of microparticles became larger, changing from 41.3 to $71.7 \mu \mathrm{m}$ (Table 1). Most of the researchers constructing TEB carriers now aim to produce nano-sized formulations, which would be able to penetrate into plant tissues [25-26]. In the present study, we propose using micro-sized TEB formulations both for preventative application and for treating spore-infected seeds by applying the fertilizer to the soil where seeds are sown. Therefore, we prepared large microparticles, whose average diameter was more than $40 \mu \mathrm{m}$.

Zeta potential of the microparticles was not influenced by TEB loading, varying between -32.6 and -35.7 $\mathrm{mV}$ (Table 1). As tebuconazole loading was increased, the yield of the microparticles with respect to the initial mass of the polymer decreased.

The presence of large TEB crystals on the surface of microparticles also increased encapsulation efficiency to $86 \%$ at $50 \%$ loading due to surface adsorption of TEB (Table 1).

Analysis of the relevant literature shows that the highest TEB EE (up to 93\%) was achieved in a study by Ding et al. [25]. The authors encapsulated TEB into self-assembled amphiphilic nanoparticles based on chitosan with branched acrylic monomers (polymethyl methacrylate and hydroxymethyl methacrylate). On the other hand, the values of EE achieved in the present study are considerably higher than the TEB EE in porous silicon nanoparticles, which was no greater than $45 \%$ [26]. Thus, polymeric carriers for TEB have better potential than inorganic ones, which is proved by their significantly higher EE.

\subsection{TEB release kinetics in vitro}

TEB release in vitro was studied in distilled water used as model medium. Figure 3 shows profiles of TEB release from $\mathrm{P} 3 \mathrm{HB}$ microparticles over two months.

We expected the greatest TEB release from the microparticles loaded at $50 \%$ of the polymer weight, as the large TEB particles present on their surface (Fig. 2) were supposed to dissolve after coming in contact with the medium, due to physical adsorption. However, the fungicide release curves obtained for the $50 \%$ and $25 \%$ loaded microparticles were similar to each other. A possible reason for this may be poor water solubility of TEB. In accordance with the theory of solubility, the chemical potentials of the solvent and solute gradually change during dissolution. Dissolution stops at a concentration at which the chemical potential of the solute in the solution is equal to the chemical potential of the dissolved substance in its pure phase. As tebuconazole is poorly dissolved in water $(<0.032 \mathrm{~g} / \mathrm{L})$, when it reached its highest possible concentration in the model medium, the rate of TEB release from the $25 \%$ and $50 \%$ loaded microparticles slowed down. 
This assumption was confirmed by results shown in Figure 3: photographs of microparticles after incubation in water. Microparticles with the highest TEB loading still had fungicide crystals on their surface, which suggested that TEB was only partly released and, hence, prolonged release of the fungicide was achieved.

After 60 days, $42.7 \pm 2.7 \%$ and $38.3 \pm 3.7 \%$ TEB was released from the particles initially loaded at $25 \%$ and $50 \%$, respectively. The amount of the fungicide released from the 10\%-loaded microparticles was the lowest $-25 \%$. The most likely causes for the slow rates of TEB release from P3HB microparticles are poor water solubility of TEB and the slow degradation rate of the polymer.

After the microparticles were incubated in water, their size distribution changed, too, and the fraction of microparticles of sizes between 100 and $150 \mu \mathrm{m}$ became larger (Fig. 4). The average diameters of the microparticles with the initial TEB loading of 10, 25, and 50\% increased to 94, 104, and $112 \mu \mathrm{m}$, respectively, as the particles formed aggregates during incubation in water.

The data obtained from in vitro TEB release were fitted to different mathematical models, namely, Zeroorder, First-order, Higuchi, Hixson-Crowell, and Korsmeyer-Pappas models, to predict the kinetics and fungicide release mechanism.

The release constant and regression coefficient $\left(\mathrm{R}^{2}\right)$ values obtained from the mathematical models are shown in Table 2 and Fig. 5.

Release kinetics of TEB from the microparticles with the lowest loading could be described using the Hixson-Crowell model with the coefficient $\mathrm{R}^{2}$ of 0.980 . The data obtained for the microparticles containing $25 \%$ and 50\% TEB best fitted the Zero-order and Higuchi models as indicated by the correlation coefficient, i.e. 0.940 , 0.989 and 0.978, 0.991, respectively, indicating that TEB release from the formulation followed Fickian diffusion. Also, the value of release exponent " $n$ " obtained by applying the Korsmeyer-Pappas equation for all formulations was below 0.5, indicating TEB release from these formulations through Fickian diffusion. Thus, the release profiles could be best explained by the Hixson-Crowell, Zero-order, and Higuchi models. microparticles.

These results suggest that fungicide release can be prolonged by varying the amount of TEB in

\subsection{Antifungal activity}

Antifungal activity of $\mathrm{P} 3 \mathrm{HB}$ microparticles loaded with the fungicide TEB was studied in experiments with phytopathogenic fungi $F$. moniliforme and $F$. solani. The reason for choosing these species was that tebuconazole most effectively inhibits biosynthesis of ergosterol in cell membranes of these phytopathogenic fungi. Photographs of phytopathogenic fungi of the genus Fusarium - F. moniliforme and F. solani-grown on solid medium in the presence of TEB-loaded microparticles can be seen in Figure 6.

The areas of the $F$. moniliforme and $F$. solani mycelial colonies in the negative control $\left(\mathrm{Control}^{-}\right)$reached 53.5 and $60.2 \mathrm{~cm}^{2}$, respectively, over 10 days. In the positive control $\left(\mathrm{Control}^{+}\right)$, the areas of the $F$. moniliforme and F. solani colonies decreased to 24.5 and $27.4 \mathrm{~cm}^{2}$, respectively (Fig. 7). The smallest areas of the colonies were observed for the fungi grown in the presence of $\mathrm{P} 3 \mathrm{HB} / \mathrm{TEB}$ microparticles. The areas of the $F$. moniliforme mycelial colonies established in the presence of microparticles loaded with $25 \%$ and $50 \%$ TEB were 18.2 and $17.7 \mathrm{~cm}^{2}$, and the areas of the F. solani mycelial colonies were 21.3 and $18.6 \mathrm{~cm}^{2}$, respectively (Fig. 7).

Thus, TEB effectively diffused from polymeric microparticles to the nutrient medium, significantly decreasing the areas of Fusarium colonies relative to the negative control.

A search of the literature revealed few studies describing development of slow-release tebuconazole formulations and evaluating their efficacy. Asrar et al. [27] described microparticles prepared from poly(methyl methacrylate) and poly(styrene-co-maleic anhydride) emulsions and loaded with TEB. The encapsulated fungicide was applied to soil and effectively controlled wheat rust Puccinia recondita. Another study described cyanobacteria cells serving as a natural environmentally friendly wall material to encapsulate TEB with urea-formaldehyde resins automatically coated on it via electrostatic interactions. A bioactivity experiment showed that encapsulated TEB authentically prolonged the antifungal effects and was very efficacious in controlling wheat powdery mildew compared with the commercial formulation [28]. Our team previously studied antifungal activity of TEB embedded in films and pellets towards $F$. moniliforme and $F$. solani. The inhibitory effect was stronger when films were used. The inhibitory effect of TEB-loaded pellets was comparable to that of the TEB commercial formulation (positive control).

Results obtained in the present study suggest effective antifungal activity of TEB encapsulated in polymeric microparticles towards $F$. moniliforme and $F$. solani.

\section{Conclusion}

TEB-loaded P3HB-based microparticles were developed and comprehensively characterized in this study. TEB-loaded microparticles were prepared using double emulsification technique. Tebuconazole encapsulation efficiency depended on the TEB to polymer matrix mass ratio, reaching 59,65, and $86 \%$ in the microparticles initially loaded with 10, 25, and 50\% TEB, respectively. As the loading amount was increased, the average diameter of microparticles increased too, from 41.3 to $71.7 \mu \mathrm{m}$, while zeta potential was not influenced by TEB loading, varying between -32.6 and $-35.7 \mathrm{mV}$. TEB release from microparticles was studied in a laboratory aqueous environment. TEB was released gradually from 10,25 , and 50\% loaded particles, and over 60 days, 25,43 , and $38 \%$, respectively, of the initially loaded amount was released. The antifungal activity of the P3HB/TEB microparticles against phytopathogenic fungi $F$. moniliforme and $F$. solani was comparable to that of the free 
fungicide. Thus, hydrophobic agrochemicals (TEB) can be effectively encapsulated into P3HB microparticles to construct slow-release formulations.

\section{Acknowledgements}

The authors would like to thank Ivan Nemtsev (Federal Research Center Krasnoyarsk Scientific Center of the Siberian Branch of the RAS) for their support with SEM.

Conflict of interest: The authors disclose that no conflicting interests associated with the manuscript exist.

\section{Funding}

This study was financially supported by Project "Agropreparations of the new generation: a strategy of construction and realization” (Agreement № 074-02-2018-328) in accordance with Resolution № 220 of the Government of the Russian Federation of April 9, 2010, "On measures designed to attract leading scientists to the Russian 
[1] Li, D.; Liu, M.; Yang, Y., Shi, H.; Zhou, J.; He, D. Strong Lethality and Teratogenicity of Strobilurins on Xenopus Tropicalis Embryos: Basing on Ten Agricultural Fungicides. Environ. Pollut. 2016, 208 (B), 868-874. https://doi.org/10.1016/j.envpol.2015.11.010

[2] Child, R.D.; Evans, D.E.; Allen, J.; Arnold G.M. Growth Responses in Oilseed Rape (Brassica Napus L.) to Combined Applications of the Triazole Chemicals Triapenthenol and Tebuconazole and Interactions with Gibberellins. Plant Growth Regul. 1993, 13, 203-212. https://doi.org/10.1007/BF00024263

[3] Montfort, F.; Klepper, B.L.; Smiley, R.W. Effects of two Triazole Seed Treatments, Triticonazole and Triadimenol, on Growth and Development of Wheat. Pestic. Sci. 1996, 46, $315-322$. https://doi.org/10.1002/(SICI)1096-9063(199604)46:4<315::AID-PS369>3.0.CO;2-R

[4] Yang, D.; Wang, N.; Yan, X.; et al. Microencapsulation of Seed-Coating Tebuconazole and its Effects on Physiology and Biochemistry of Maize Seedlings. Coll. Surf. B Biointer. 2014, 114, $241-246$. https://doi.org/10.1016/j.colsurfb.2013.10.014

[5] Grossmann, K. Plant Growth Retardants as Tools in Physiological Research. Physiol. Plant. 1990, 78, 640-648. https://doi.org/10.1111/j.1399-3054.1990.tb05254.x

[6] Fletcher, R.A.; Gilley, A.; Sankhla N.; Davis, T.D. Triazoles as Plant Growth Regulators and Stress Protectants. Horticultural Reviews. 2000, 24, 55-138. https://doi.org/10.1002/9780470650776.ch3

[7] Berenzen, N.; Lentzen-Godding, A.; Probst, M.; Schulz, H.; Schulz, R.; Liess, M. A Comparison of Predicted and Measured Levels of Runoff-Related Pesticide Concentrations in Small Lowland Streams on a Landscape Level. Chemosphere. 2005, 58, 683-691. https://doi.org/10.1016/j.chemosphere.2004.05.009

[8] Reilly, T.J.; Smalling, K.L.; Orlando, J.L.; Kuivila, K.M. Occurrence of Boscalid and Other Selected Fungicides in Surface Water and Groundwater in Three Targeted Use Areas in the United States. Chemosphere. 2012, 89, 228-234. https://doi.org/10.1016/j.chemosphere.2012.04.023

[9] Ghormade, V.; Deshpande, M.V.; Paknikar, K.M. Perspectives for Nano-Biotechnology Enabled Protection and Nutrition of Plants. Biotechnol. Adv. 2011, 29, 792-803 https://doi.org/10.1016/j.biotechadv.2011.06.007

[10] Alves, V. D.; Torres, C. V.; Freitas, F. Bacterial Polymers as Materials for the Development of Micro/Nanoparticles. Int. J. Polym. Mat. Polym. Biomater. 2016, 65, $211-224$. http://dx.doi.org/10.1080/00914037.2015.1103239

[11] Singh, B.; Sharma, D. K.; Gupta A. The controlled and sustained release of a fungicide from starch and alginate beads. J. Env. Sci. Health B, 2009, 44(2), 113-122. https://doi.org/10.1080/03601230802599019

[12] Lobo, F.; Aguirre, C.; Silva, M.; Grillo, R.; de Melo, N.; Oliveira, L.; Morais, L.; Campos, V.; Rosa, A.; Fraceto, L. Poly(hydroxybutyrate-cohydroxyvalerate) Microspheres Loaded with Atrazine Herbicide: Screening of Conditions or Preparation, Physico-Chemical Characterization, and In Vitro Release Studies. Polym. Bull. 2011, 67, 479-495. doi:10.1007/s00289-011-0447-6

[13] Gimeno M. An overview of the latest development of microencapsulation for agricultural products. J. Env. Sci. Health B, 1996, 31(3), 407-420. https://doi.org/10.1080/03601239609373001

[14] Zhila, N.; Murueva, A.; Shershneva, A.; Shishatskaya, E.; Volova, T. Herbicidal activity of slowrelease herbicide formulations in wheat stands infested by weeds. J. Env. Sci. Health B, 2017, 52(10), 729-735 https://doi.org/10.1080/03601234.2017.1356668

[15] Wang, Y.; Qian, C.; Yan, X.; Liu, H. Preparation and Characterization of Controlled Release Poly(melamine-Formaldehyde) Microcapsules Filled with 2,4-D Isooctyl Ester. Int. J. Polym. Mat. Polym. Biomater. 2017, 66, 963-969. https://doi.org/10.1080/00914037.2017.1291511

[16] Pascoli, M.; Lopes-Oliveira, P. J.; Fraceto, L. F .; Barozzi, S. A.; Oliveira, H. C. State of the Art of Polymeric Nanoparticles as Carrier Systems with Agricultural Applications: a Minireview. Energ. Ecol.Environ. 2018, 3, 137-148. https://doi.org/10.1007/s40974-018-0090-2

[17] Volova, T.G.; Shishatskaya, E.I.; Sinskey, A.J. Degradable Polymers. Production, Properties and Applications, Nova Science Publishers: New York, 2013.

[18] Grillo, R.; Pereira, A. E. S.; Melo, N.F.S.; Portoc, R. M.; Feitosa, L. O.; Tonello, P.S.; Filho, N. L. D.; Rosa, A. H.; Limac, R.; Fraceto, L. F. Controlled Release System for Ametryn Using Polymer Microspheres: Preparation, Characterization and Release Kinetics in Water. J. Hazardous Mater. 2011, $186,1645-1651$. doi:10.1016/j.jhazmat.2010.12.044

[19] Suave, J.; Dall'Agnol, E. C.; Pezzin, A. P. T.; Meier, M. M.; Silva, D. A. K. Biodegradable Microspheres of Poly(3-hydroxybutyrate)/Poly(e-caprolactone) loaded with Malathion Pesticide: Preparation, Characterization, and In Vitro Controlled Release Testing. J. App. Polym. Sci. 2010, 117, 3419-3427. https://doi.org/10.1002/app.32082

[20] Volova, T. G.; Prudnikova, S. V.; Zhila, N. O.; Vinogradova, O. N.; Shumilova, A. A.; Nikolaeva, E. D.; Kiselev E. G.; Shishatskaya E. I. Efficacy of Tebuconazole Embedded in Biodegradable Poly-3hydroxybutyrate to Iinhibit the Development of Fusarium moniliforme in Soil Microecosystems. Pest. Manag. Sci. 2017, 73, 925-935. https://doi.org/10.1002/ps.4367

[21] Volova, T.G.; Prudnikova S.V.; Zhila N.O. Fungicidal Activity of Slow-Release P(3HB)/TEB Formulations in Wheat Plant Communities Infected by Fusarium moniliforme. Environ. Sci. Pollut. Res. 2018, 25, 552-561. doi: 10.1007/s11356-017-0466-4 
[22] Volova, T.G.; Gitelzon, I.I; Kalacheva, G.S.; Kuznrtsov, V.N.; Shabanov, V.F. Means of obtaining a polymer of $\beta$-oxybutyric acid. Patent of Russian Federation for invention, № 2207375, 2001.

[23] Volova, T. G.; Zhila, N. O.; Vinogradova, O. N.; Nikolaeva, E.D.; Kiselev, E.G.; Shumilova, A. A.; Shershneva, A. M.; Shishatskaya E.I. Constructing Herbicide Metribuzin Sustained-Release Formulations Based on the Natural Polymer Poly-3-hydroxybutyrate as a Degradable matrix. J. Env. Sci. Health B, 2016, 51, 113-125 http://dx.doi.org/10.1080/03601234.2015.1092833

[24] Nasir, F.; Iqbal, Z.; Khan, J.A.; Khan, A.; Khuda, F.; Ahmad, L.; Khan, A.; Khan, A.; Dayoo A. Development and evaluation of diclofenac sodium thermorevesible subcutaneous drug delivery system. Int. J. Pharmaceut. 2012, 439 (1-2), 120-126. https://doi.org/10.1016/j.ijpharm.2012.10.009

[25] Ding, X.; Richter, D.L.; Matuana, L.M.; Heiden, P.A. Efficient One-Pot Synthesis and Loading of Self-Assembled Amphiphilic Chitosan Nanoparticles for Low-Leaching Wood Preservation. Carbohydr. Polym. 2011, 86, 58-64. https://doi.org/10.1016/j.carbpol.2011.04.002

[26] Qian, K.; Shi, T.; He, S. Release Kinetics of Tebuconazole from Porous Hollow Silica Nanospheres Prepared by Miniemulsion Method. Micropor. Mesopor. Mater. 2013, 169, 1-6. https://doi.org/10.1016/j.micromeso.2012.10.017

[27] Asrar, J.; Ding, Y.; Monica, R.E. La.; Ness, L.C. Controlled Release of Tebuconazole from a Polymer Matrix Microparticle: Release Kinetics and Length of Efficacy. J. Agric. Food Chem. 2004, 52, 4814-4820 doi:10.1021/jf0306385

[28] Zhang, B.; Zhang, T.; Wang, Q.; Ren, T. Microorganism-Based Monodisperse Microcapsules: Encapsulation of the Fungicide Ttebuconazole and its Controlled Release Properties. RSC Adv. 2015, 5, 2516425170. doi: 10.1039/c5ra01629k 


\section{Figure caption}

Figure 1. Structural formulas of poly-3-hydroxybutyrate (a) and tebuconazole (b)

Figure 2. SEM images of P3HB/TEB microparticles with different TEB loading (10, 25, and 50\% of the polymer weight) and their size distribution: 1, 2, and 3, respectively. Bars 500, 40, and $2 \mu \mathrm{m}$

Figure 3. TEB release kinetics from P3HB microparticles loaded with 10, 25, and 50\% TEB (relative to the polymer weight) for 60 days of incubation in distilled water. Bars 500, 40, and $2 \mu \mathrm{m}$

Figure 4. SEM images of P3HB/TEB microparticles with different TEB loading - 10, 25, and 50\% of the polymer weight: 1, 2, and 3, respectively, after 60 days of incubation in distilled water. Bars 500, 40, and $2 \mu \mathrm{m}$

Figure 5. Mechanism of TEB release: by Zero-order model; by First-order model; by Higuchi model; by Hixson-Crowell model

Figure 6 - Sensitivity of Fusarium species to TEB: 1) P3HB microparticles loading $25 \%$ TEB; 2) P3HB

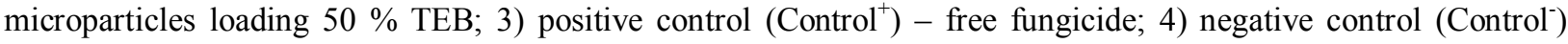
without TEB

Figure 7 - The petri images of changes in the area of the Fusarium mycelial colonies affected by free TEB $\left(\mathrm{Control}^{+}\right)$and TEB encapsulated in $\mathrm{P} 3 \mathrm{HB}$ microparticles containing $25 \mathrm{mg}$ TEB and $50 \mathrm{mg}$ TEB. $\mathrm{Control}^{-}$is negative control, without TEB 